\title{
On the crosswind stability of high speed railway vehicles
}

\author{
Christian Wetzel* and Carsten Proppe \\ Institut für Technische Mechanik, Universität Karlsruhe (TH), 76128 Karlsruhe, Germany
}

\begin{abstract}
The crosswind stability against overturning is a major national design criterium for high-speed railway vehicles. Due to the increasing interoperability in Europe it has also become an important international task. Especially modern light-weight trains are at risk to fail and counter-measures as for example wind-fences or adding extra weight in the underbelly are always very expensive. In recent years efforts were made to derive an uniform rule in certifying railway vehicles. In this case especially probabilistic methods were proposed. These probabilistic technics are common design criteria for wind turbines. This paper presents a sophisticated method to compute the reliability of railway vehicles under strong crosswind. In consideration of the given gust signal and the high-frequency, turbulent fluctuations of the wind the response of a simplified train model is computed. The major failure criterium to determine the reliability is the lowest wheel-rail contact force of the railway vehicle.
\end{abstract}

(C) 2006 WILEY-VCH Verlag GmbH \& Co. KGaA, Weinheim

\section{Introduction}

The intention of this paper is to present a method to calculate the probability of failure $P_{f}$ of a high-speed railway vehicle under strong crosswind. In this case failure means the exceedance of a critical value of the so-called wheel unloading. The wheel unloading is defined as $\frac{\delta Q}{Q}=1-\frac{Q_{\text {dyn }}}{Q_{\text {static }}}$, where $Q_{\text {dyn }}$ is the wheel-rail contact force in every time step and $Q_{\text {static }}$ is the static contact force [3]. For the entire system a statistical analysis is needed because of the stochastic properties of the excitations [1]. There are three important stochastic influences. The wind excitation is a stochastic process, the aerodynamic coefficients $C_{(\text {side, lift, roll, pitch, yaw) }}$ are uncertain and the track has stochastic irregularities.

\section{Modelling of the system}

For such a complex system with multi degrees of freedom and sophisticated wheel-rail contacts it is almost impossible to derive the equations of motion in an analytical form. Because of this the railway vehicle is simulated in the commercial MBS-Code ADAMS/RAIL. The wind-forces and the wind-moments are modeled as concentrated forces and moments which are acting on the centre of mass of the vehicle [3]. For the calculation of these forces and moments the density of the air $\rho_{L}$, the height $h$, the width $w$ and the length $l$ of the railway vehicle are needed.

$$
\begin{aligned}
F_{y}\left(v_{o}, v(t)\right) & =C_{\text {side }}(\beta) \frac{\rho_{L} h l}{2} v_{s}^{2}, F_{z}\left(v_{o}, v(t)\right)=C_{\text {lift }}(\beta) \frac{\rho_{L} w l}{2} v_{s}^{2}, M_{x}\left(v_{o}, v(t)\right)=C_{\text {roll }}(\beta) \frac{\rho_{L} l h^{2}}{2} v_{s}^{2}, \\
M_{y}\left(v_{o}, v(t)\right) & =C_{\text {pitch }}(\beta) \frac{\rho_{L} w l^{2}}{2} v_{s}^{2}, M_{z}\left(v_{o}, v(t)\right)=C_{\text {yaw }}(\beta) \frac{\rho_{L} h l^{2}}{2} v_{s}^{2}, \\
\beta & =\arctan \left(\frac{v(t)}{v_{o}}\right), v_{s}=\sqrt{v^{2}(t)+v_{o}^{2}} . \quad \text { Crosswind }
\end{aligned}
$$

a)

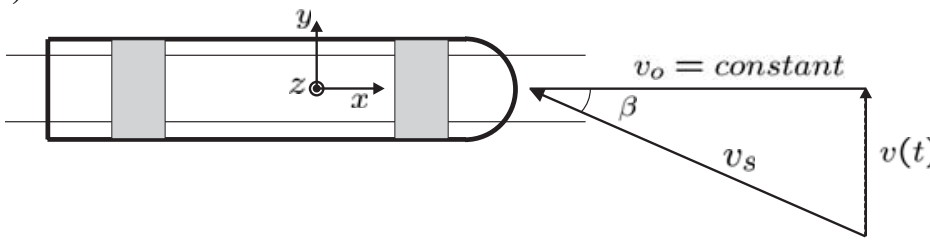

b)

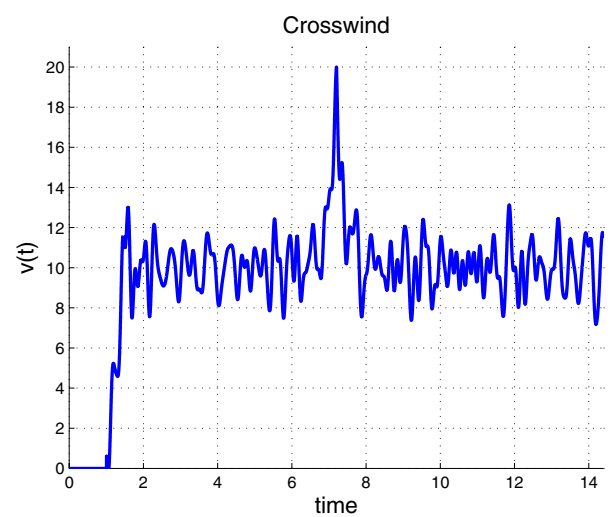

Fig. 1 a) Sketch of the system, b) Characteristic of the crosswind

* Corresponding author: e-mail: wetzel@itm.uni-karlsruhe.de, Phone: +49 721 608 6823, Fax: +497216086070 
The magnitudes of the forces, of the moments and of the aerodynamic coefficients depend on the velocity of the crosswind $v(t)$ and on the driving velocity $v_{o}$ of the railway vehicle.

\section{Modelling of the windloading}

The model for the crosswind consists of a superposition of the mean wind, the gust characteristic and the turbulent fluctuations as shown in figure 1b). The mean wind velocity and the gust characteristic are modeled according to a proposal of the DeuFraKo-Project [4]. The superposed turbulent fluctuations $v^{\prime}(t)$ are computed by a spectral decomposition of the "VonKarman"-spectral density function $S(f)$ which is often used to describe turbulent flows [2]:

$$
S(f)=\frac{4 x \sigma_{u}^{2}}{\left.f\left(1+70.8 x^{2}\right)^{\frac{5}{6}}\right)}, \quad x=\frac{f L_{u x}}{u_{o}}, \quad v^{\prime}(t)=\sum_{k=1}^{n} \sqrt{S\left(f_{k}\right) f_{o}}\left(A_{k} \cos \left(2 \pi f_{k} t\right)+B_{k} \sin \left(2 \pi f_{k} t\right)\right) .
$$

In the equations (2) the term $L_{u x}$ is a function of the surface roughness, $f_{o}$ is the fundamental frequency and the standard normal variables $A_{k}$ and $B_{k}$ describe the stochastic characteristic of the turbulent fluctuations $v^{\prime}(t)$. The whole set of the stochastic variables of the system consist of the gust-amplitude $A$, the gust-length $L$, the aerodynamic coefficients $C_{(\ldots)}$ and the coefficients $A_{k}, B_{k}$ of the turbulence.

\section{Statistical description of the system}

For the calculation of the probability of failure $P_{f}$ it is necessary to evaluate the high dimensional integral

$$
P_{f}=\int_{\Omega_{f}} p_{z^{*}}\left(\underline{z}^{*}\right) d \underline{z}^{*}
$$

over the failure domain $\Omega_{f}$ whereas $\underline{z}^{*}$ is the array of all stochastic variables and $p_{z^{*}}\left(\underline{z}^{*}\right)$ are the corresponding probability density functions. The probability of failure can be computed by using the law of conditional probability. The probability of failure $P_{f}$ is approximated by the conditional probability

$$
P_{f}=\int_{u_{o, d}}^{u_{o, t}} P\left(\underline{z} \mid u_{o}\right) p\left(u_{o}\right) d u_{o} \approx \sum_{l=1}^{N} P\left(\underline{z} \mid u_{o_{l}}\right) p\left(u_{o_{l}}\right) \Delta u_{o}, \underline{z}=\left[A, L, C_{(\ldots)}, A_{k}, B_{k}\right]
$$

wherefor an integration over the mean wind velocity $u_{o}$ has to be done. The major task in this case is the computation of the conditional probability $P\left(\underline{z} \mid u_{o}\right)$ as the dimension of this integral is very high and so classical numerical routines of integration cannot be used. Furthermore the failure domain is not known explicitly but can only be computed pointwise by a numerical simulation. Due to these restrictions the failure integral is approximated by introducing an estimator $\bar{P}\left(\underline{z} \mid u_{o}\right)$ and using Monte Carlo Simulation.

$$
\bar{P}\left(\underline{z} \mid u_{o}\right)=\frac{1}{N} \sum_{i=1}^{N} \mathrm{I}(g(\underline{z})), \quad \mathrm{I}(g(\underline{z}))=\left\{\begin{array}{ll}
1, & \text { if } g(\underline{z}) \leq 0 \\
0, & \text { if } g(\underline{z})>0
\end{array}, \quad g(\underline{z})=0.9-\max \left(\frac{\delta Q}{Q}\right)\right.
$$

The so-called limit-state function $g(\underline{z})=0$ separates the safe domain from the failure domain. The wheel unloading function $\frac{\delta Q}{Q}$ does not exist in an analytical form. As the the failure domain is only known pointwise by a numerical simulation of the system an indicator function $\mathrm{I}(g(\underline{z}))$ is introduced as shown in equations (5). A major drawback in using Monte Carlo Simulations is the huge amount of independent simulations which have to be done to get a reasonable accuracy. The computation time rises up to a such a long time span (one month and more) that the method is not feasible anymore. The numerical Monte Carlo computations of the probability of failure will be improved by using a FORM analysis and by constructing an importance sampling density.

\section{References}

[1] A. Carrarini, A probabilistic approach to the effects of cross-winds on rolling stock. Proc. European Congress on Computational Methods in Applied Sciences and Engineering. Jyväskylä (2004).

[2] W. Bierbooms, P.-W. Cheng, Stochastic gust model for design calculations of wind turbines. Wind Engineering and Industrial Aerodynamics 90, 1237-1251 (2002).

[3] B. Diedrichs et al., Quasi-static modelling of wheel-rail reactions due to crosswind effects for various types of high-speed rolling stock. Proceedings of the I MECH E Part F Journal of Rail and Rapid Transit, No. F2 16, 133-148 (2004).

[4] DeuFraKo-Projekt, Seitenwindverhalten von Schienenfahrzeugen. DB AG, DB Systemtechnik (2003). 